\title{
PINP as a biological response marker during teriparatide treatment for osteoporosis
}

\author{
J. H. Krege • N. E. Lane • J. M. Harris • P. D. Miller
}

Received: 1 October 2013 / Accepted: 4 February 2014 / Published online: 6 March 2014

(C) The Author(s) 2014. This article is published with open access at Springerlink.com

\begin{abstract}
Postmenopausal women with severe osteoporosis may require treatment with the bone anabolic drug teriparatide. While changes in bone mineral density (BMD) are one measure of response, BMD changes often require a minimum of one year to observe measureable changes. Biochemical markers of bone turnover change within 1 to 3 months of initiating osteoporosis therapy. Monitoring with a marker such as procollagen type I N propeptide (PINP), an osteoblast-derived protein, during teriparatide treatment may provide clinically useful information for managing patients with osteoporosis. Clinical trials have shown consistent increases in PINP within 3 months of initiating teriparatide, increases that are significantly greater than placebo and significantly different from baseline. Increases in PINP concentrations during teriparatide treatment correlate well with increases in skeletal activity assessed by radioisotope bone scans and quantitative bone histomorphometry parameters. Individuals treated with teriparatide in clinical trials usually experienced an increase in PINP $>10 \mathrm{mcg} / \mathrm{L}$ from baseline, while those given placebo usually did not. In the
\end{abstract}

\section{J. H. Krege}

Lilly USA, Indianapolis, IN, USA

\section{N. E. Lane}

Department of Medicine and Center for Musculoskeletal Health, University of California at Davis, Sacramento, CA, USA

J. M. Harris

Eli Lilly and Company, Indianapolis, IN, USA

P. D. Miller

Department of Medicine, University of Colorado Health Sciences

Center, Denver, CO, USA

P. D. Miller $(\bowtie)$

Colorado Center for Bone Research, 3190 S. Wadsworth Boulevard,

Lakewood, CO 80227, USA

e-mail: millerccbr@aol.com clinical setting, patients experiencing a significant increase in PINP $>10 \mathrm{mcg} / \mathrm{L}$ after initiating teriparatide therapy may receive an earlier confirmation of anabolic effect, while those who do not may be assessed for adherence, proper injection technique, or undetected secondary conditions that might mitigate an anabolic response. PINP monitoring may provide information supplemental to BMD monitoring and be a useful aid in managing patients receiving anabolic osteoporosis treatment in the same way that biochemical markers of bone resorption are useful in monitoring antiresorptive therapy. This review examines PINP as a biological response marker during teriparatide treatment for osteoporosis.

Keywords Anabolics · Biochemical markers of bone turnover · Osteoporosis · PINP · Teriparatide

\section{Introduction}

Patients with osteoporotic fractures may be asymptomatic and yet have significant skeletal deterioration and high risk for future fractures. In such patients, teriparatide has the potential to increase bone formation on bone surfaces, increase bone mineral density (BMD), increase bone strength, and reduce the risk of vertebral and nonvertebral fractures [1-7].

After a patient is prescribed teriparatide, initiating and continuing treatment to obtain a biological response requires several steps: (1) the patient must acquire the medication and supplies for subcutaneous (SC) injections; (2) the patient must refrigerate the medication at $2-8^{\circ} \mathrm{C}$ to retain its stability [8]; (3) the patient must self-administer teriparatide properly by daily SC injection or make arrangements for a trained caregiver to administer the injection [8]; (4) the patient must not have medical conditions that preclude an anabolic response [8]; and (5) the patient must persist with treatment to achieve the desired response. Assessing all these steps through a 
detailed patient interview might be difficult to accomplish. In addition, prescribers may overestimate their patients' adherence to treatment. For example, Curtis and colleagues [9] reported physicians estimated $67.2 \%$ of their osteoporosis patients to be adherent, while only $40 \%$ of patients were actually adherent based on pharmacy data.

Concurrent monitoring for a biological response to teriparatide may provide useful clinical information for the prescriber and for the patient with osteoporosis at high risk for fracture. However, BMD testing is typically not obtained until after 1 to 2 years of treatment $[10,11]$. This delay in assessing for biological response may be problematic in light of a need for bone formation in patients at high risk for fracture and a 2year lifetime exposure limitation to teriparatide [8]. Accordingly, a biological response marker measureable earlier than BMD may be useful to more proactively manage patients treated with teriparatide.

Biochemical markers of bone turnover have the potential to provide early feedback to patients and prescribers during osteoporosis treatments [12-14]. For example, patients treated with teriparatide exhibiting a positive biochemical marker response can receive confirmation of an anabolic biologic response in the bone. This may be especially important since overall patient compliance with osteoporosis treatments is inadequate and could be enhanced by the assurance of a positive biological response to the medication [15]. In fact, the International Osteoporosis Foundation (IOF) determined the most common factor women cite for persisting with their osteoporosis treatment: "knowing I'm doing something to help" [16]. On the other hand, patients without a significant change in biochemical marker concentration during teriparatide treatment can be assessed for difficulties with injection technique, medication storage, adherence, or for conditions that might impair response to therapy. If problems are identified, addressed, and corrected, this type of early assessment has the potential to improve patient outcome [17].

Several biochemical markers of bone formation may be measured in the serum: osteocalcin, bone-specific alkaline phosphatase (bone ALP), procollagen type I $\mathrm{N}$ propeptide (PINP), and procollagen type I C propeptide (PICP) [12, 14, 18]. Because type I collagen is the most prevalent protein in bone, measuring byproducts of collagen synthesis is an appealing approach. Following the synthesis of new type I collagen within the osteoblast, PINP is cleaved from type I procollagen by proteases outside the osteoblast (Fig. 1) [12, 18]. Serum PINP concentrations reflect the integrated amount of skeletal new bone formation [19]. Thus, diseases involving high bone turnover would be expected to be associated with high serum concentrations of PINP; examples are metabolic bone diseases including osteomalacia and Paget's disease, endocrine disorders including thyrotoxicosis and primary hyperparathyroidism, and malignant bone disease such as
Bone Collagen Synthesis

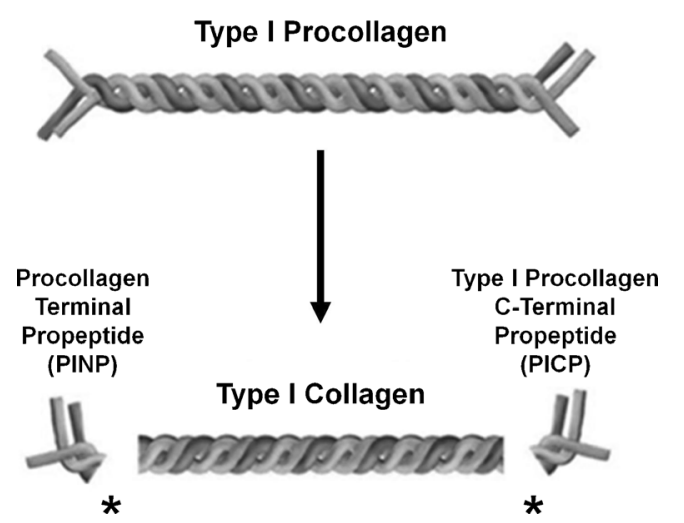

Fig. 1 Type 1 collagen formation and procollagen type I N propeptide $(P I N P)$. Type 1 procollagen is made in the osteoblast and secreted into new bone matrix. In the bone matrix, procollagen peptidases cleave PINP from the amino $(\mathrm{N})$ terminal end and procollagen type I C propeptide $(P I C P)$ from the carboxy $(\mathrm{C})$-terminal end of type 1 procollagen, resulting in mature type 1 collagen. Mature type 1 collagen is the most common protein in bone; a triple helical structure composed of two alpha-1 chains and one alpha- 2 chain. PINP and PICP make their way into the circulation. Within the circulation, PINP exists as different linear forms including the original intact trimeric PINP and the monomeric or dimeric degradation products. Available PINP assays measure either intact triple helical PINP or total PINP, which includes intact PINP as well as the other forms of PINP. With permission from Bauer et al. [13]

multiple myeloma [12]. Importantly, the label warns against the use of teriparatide in patients with these diseases [8].

While PINP primarily originates from bone, small amounts of type I collagen, and hence PINP, are contributed from other tissues such as skin, tendon, dentin, ligaments, cartilage, and interstitial tissues [14, 20]. For example, the acute fibroproliferative reaction in a healing wound is associated with increased concentrations of PINP in the extra-cellular fluid of the wound [21].

Serum PINP monitoring may be particularly clinically useful because this marker is relatively insensitive to food and circadian rhythm effects, and in clinical trials, this marker has been shown to increase dynamically in response to teriparatide treatment $[17,18,22-25]$. The IOF and the International Federation of Clinical Chemistry (IFCC) have recently published an extensive review of bone turnover markers and recommend PINP as the reference biochemical marker of bone formation [12]. Likewise, the National Bone Health Alliance has also recommended PINP as the reference marker for bone formation [13].

The purpose of this review is to evaluate PINP as a biological response marker during teriparatide treatment for osteoporosis. A useful classification of biomarkers developed by the Food and Drug Administration (FDA) is shown in Table 1 [26]. While some data suggest bone turnover markers might be used in the future as a component of surrogate end points for bone strength improvements achieved by pharmacotherapeutics, more data are needed before this 
Table 1 Categorization of biomarkers by the US Food and Drug Administration (FDA) [26]

\begin{tabular}{cl}
\hline Biomarker category & Characteristics \\
\hline Prognostic biomarker & $\begin{array}{c}\text { Categorizes patients by probability for disease } \\
\text { occurrence or progression in the absence of } \\
\text { intervention }\end{array}$ \\
$\begin{array}{c}\text { Categorizes patients by their probability for a } \\
\text { positive or negative response to a treatment }\end{array}$ \\
$\begin{array}{c}\text { Pemonstrates biological response has occurred } \\
\text { biomarker }\end{array}$ & $\begin{array}{c}\text { in a patient after having received a therapeutic } \\
\text { intervention }\end{array}$ \\
$\begin{array}{c}\text { Surrogate end point } \\
\text { biomarker }\end{array}$ & $\begin{array}{c}\text { Substitutes for a clinical efficacy end point and } \\
\text { is expected to predict clinical benefit or harm }\end{array}$ \\
& $\begin{array}{c}\text { Requires robust scientific evidence to justify } \\
\text { qualification as a surrogate end point }\end{array}$ \\
\hline
\end{tabular}

practice gains wide acceptance [27]. In particular, designating PINP as a surrogate end point for fracture risk reduction is not possible since data proving a definitive link between changes in PINP during teriparatide treatment to fracture risk reduction are not yet available [27]. This review will focus on PINP as a pharmacodynamic marker and will subsequently refer to PINP as a biological response marker of bone formation.

\section{Methods}

Based on a review of the literature, studies involving individuals treated with teriparatide $20 \mathrm{mcg}$ /day by SC injection with a measured assessment of PINP during treatment were included for this review. This review summarizes published findings and new and previously unpublished data, which are noted as such.

Sample collection and logistics

Commercially available PINP assays differ by the form of PINP they measure. The intact PINP assay measures the intact aminoterminal propeptide form of type 1 collagen (triple helical or trimeric), while the total PINP assay measures the intact propeptide form (trimeric) as well as smaller monomeric byproducts of PINP degradation [18]. Assays for measuring intact PINP include a radioimmunoassay (RIA) for intact PINP (UniQ ${ }^{\mathrm{TM}}$, Orion Diagnostica, Espoo, Finland) [19] and the IDS-iSYS ${ }^{\text {TM }}$ Intact PINP assay, an automated chemiluminescence immunoassay (CLIA; Immunodiagnostics Systems, Scottsdale, AZ, USA) [28]. Options for measuring total PINP include an automated electrochemiluminescence immunoassay (ECLIA) for total PINP (Roche Diagnostics, Indianapolis, IN, USA) [29] and an enzyme-linked immunosorbent assay (ELISA) for total PINP (USCN Life Science, Inc., Houston, TX,
USA) [30, 31]. For healthy individuals, there appears to be little difference between the results obtained for intact and total PINP measurements [31]. However, in the presence of reduced renal function, the clearances of intact and total PINP may differ [18]. Intact PINP relies on the liver for clearance, while the monomeric form of PINP, a component of total PINP, relies on kidney function for clearance, thereby making the interpretation of the total PINP assays potentially difficult in the setting of kidney disease [31, 32].

In the USA, the Orion UniQ ${ }^{\mathrm{TM}}$ PINP assay is currently the only FDA-approved intact PINP assay [12, 19, 29, 31]. The IDS intact PINP assay shows good agreement with the Orion assay [28, 33, 34]. In many geographies, the Roche total PINP assay is approved for clinical use [12]. This review includes data demonstrating the effect of teriparatide treatment on PINP measured by the Roche total PINP assay and by the UniQ $^{\mathrm{TM}}$ intact PINP assay. In general, the results appear to be similar.

According to the UniQ ${ }^{\mathrm{TM}}$ kit insert, no special preparation of the patient, such as fasting, prior to sample collection is needed [19]. The test may be run on serum from a venous blood sample collected by standard venipuncture technique. After specimen collection, the blood is allowed to clot and the serum is separated by centrifugation. Serum samples may be stored prior to PINP analysis for up to 5 days at 2 to $8^{\circ} \mathrm{C}$ and stored for longer periods at $-20{ }^{\circ} \mathrm{C}$ or lower [19]. Repeated freezing and thawing of samples should be avoided [19]. However, different patient preparation and sample-handling directions have also been discussed. For example, using plasma samples may be preferable to serum for PINP analysis [13].

\section{Results}

Evidence supporting PINP as a biological response marker during teriparatide treatment

The teriparatide Phase 3 Fracture Prevention Trial (NCT00670501) was a randomized, placebo-controlled trial of postmenopausal women with osteoporosis and vertebral fractures [5]. The prospectively collected biological markers of bone formation included carboxy-terminal extension peptide of procollagen type 1 (PICP) and serum bone-specific alkaline phosphatase (bone ALP). In the teriparatide group, PICP increased dynamically at 1 month but returned toward baseline by 3 months [24]. The transience of PICP elevation during teriparatide treatment suggests it is not an optimal biological response marker. In contrast, bone ALP gradually increased between baseline and 12 months in the teriparatide group [24]. Yet, because bone ALP does not show dynamic increases early during teriparatide treatment, it also may not be 
optimal as an early biological response marker in clinical practice.

Following completion of this trial, intact PINP was used to measure serum PINP concentrations from samples collected in a subset of patients at baseline and after 3 months on study drug [24]. These samples had been stored for at least 4 years and previously thawed at least twice prior to PINP assessment [17]. In a subsequent analysis of these trial results, the PINP response to teriparatide (signal) was found to be large compared to the variability (noise, or fluctuation, with placebo) of the test, as shown in Fig. 2 [17]. A relatively high signal-tonoise ratio of PINP relative to other prospectively defined markers of bone turnover was observed in the trial [17]. Accordingly, PINP may be particularly useful as a biological response marker in patients treated with teriparatide.

Subsequent teriparatide clinical trials have included prospectively collected PINP serum samples within their study design. For example, in a global, randomized, double-blind comparator trial of teriparatide vs. alendronate in 203 postmenopausal women with osteoporosis, intact PINP was assessed at baseline, 1, 3, 6, and 12 months of treatment [35]. Figure 3 shows changes in PINP concentration and urinary N-terminal cross-linking telopeptide of type I collagen corrected for creatinine (NTX-I; biochemical marker of bone resorption) in patients treated with teriparatide [35]. Following initiation of teriparatide, PINP concentration increased rapidly, with an increase significantly greater than baseline, and then further increased to remain significantly above baseline at 6 months $(p<0.010)$ [35]. Yet, the resorption marker NTX-I did not increase at 1 month but did increase after 3 months of teriparatide therapy [35]. In contrast, PINP and NTX-I concentrations decreased markedly during alendronate treatment [35]. Similarly, in another active comparator study of 44 postmenopausal women, PINP increased in patients treated with teriparatide and decreased in patients treated with risedronate, as measured by total PINP assay [36]. In addition, another active comparator study of 79 postmenopausal women demonstrated marked increases in total PINP concentration from baseline in women treated with teriparatide, with slight decreases in PINP from baseline during strontium ranelate therapy (NCT00239629) [37]. These observed changes in serum PINP concentration are consistent with the anabolic mechanism of action of teriparatide.

Changes in PINP concentration were evaluated during a Phase 3 study of teriparatide conducted in Japanese men and postmenopausal women with osteoporosis at high risk for fracture (NCT00433160) [38]. This double-blind, placebocontrolled trial randomized patients $2: 1$ to teriparatide $(N=$ $137)$ or placebo $(N=70)$ and included prospectively defined intact PINP assessments at baseline, 1, 3, 6, and 12 months of treatment. Fig. 4 shows the effects of teriparatide vs. placebo on median PINP concentrations in this study population [39]. These changes in serum PINP concentration were significantly different at $1,3,6$, and 12 months $(p<0.001)$ [39]. The sharpest rise in PINP concentration occurred within the first month of teriparatide treatment [39].

Patients treated with teriparatide may have been treated previously with antiresorptive drugs and switched to

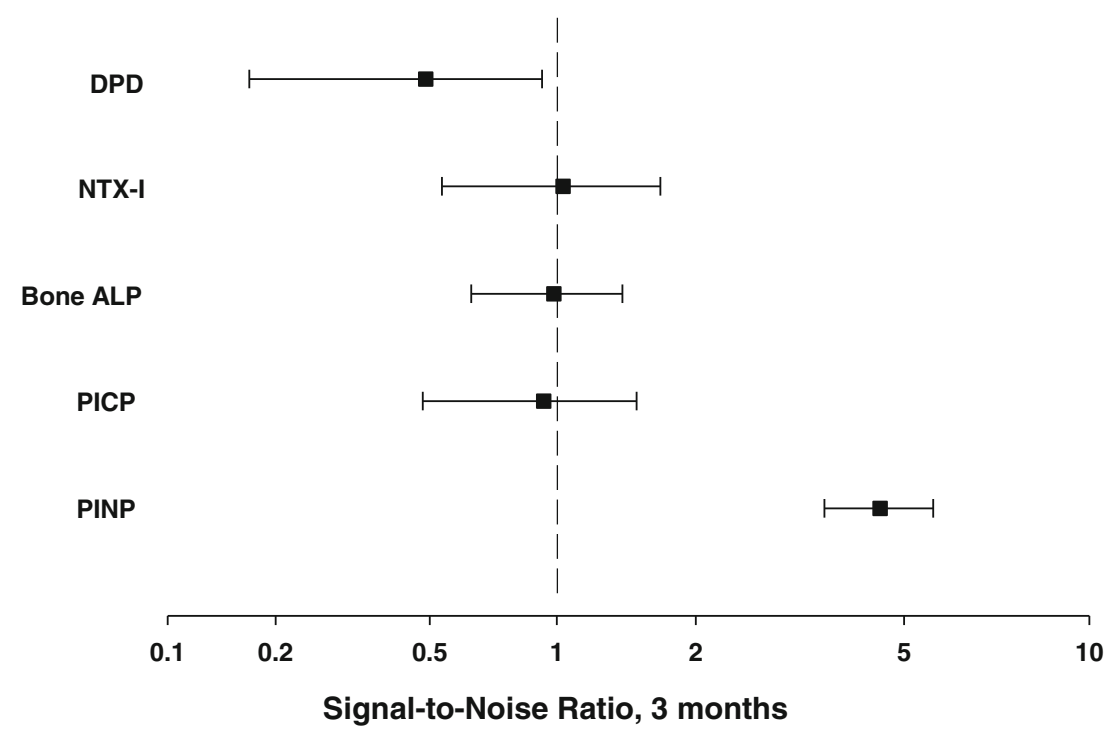

Fig. 2 Signal-to-noise ratio for biological markers of bone turnover measured at 3 months in the Fracture Prevention Trial of postmenopausal women with osteoporosis. Bone turnover markers other than PINP were measured in a subset of 171 women in the teriparatide $20 \mathrm{mcg} /$ day group and 175 women in the placebo group. PINP was measured in a subset of 254 women in the teriparatide $20 \mathrm{mcg} / \mathrm{day}$ group and 260 women in the placebo group from samples that had been stored frozen for at least

4 years and had been previously thawed at least twice [17]. DPD urinary deoxypyridine corrected for creatinine, $N T X-I$ urinary N-terminal crosslinking telopeptide of type I collagen corrected for creatinine, Bone ALP bone alkaline phosphatase, $P I C P$ procollagen type I C propeptide, PINP intact procollagen type I $\mathrm{N}$ propeptide. Adapted from Eastell and colleagues [17] 
Fig. 3 Typical effects of teriparatide on bone formation and bone resorption markers during teriparatide treatment in postmenopausal women with osteoporosis. Percentage change (adjusted least squares mean \pm standard error) from baseline in serum PINP and urinary NTX-I in patients treated with teriparatide. PINP intact procollagen type I N propeptide, NTX-I urinary Nterminal cross-linking telopeptide of type I collagen corrected for creatinine. ${ }^{*} p<0.001,{ }^{* *} p<0.050$ for change from baseline within-group comparisons. Adapted from McClung and colleagues [35]

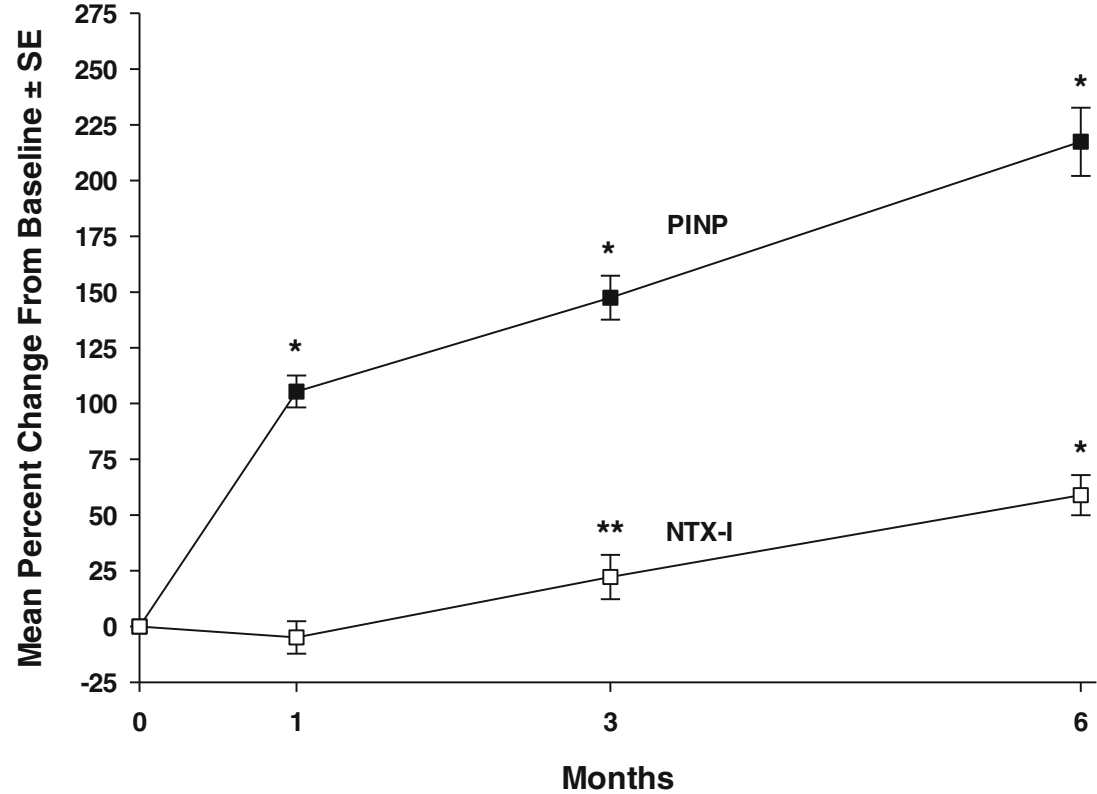

teriparatide. In the EUROFORS study, 758 postmenopausal women with osteoporosis who were either treatment-naive, previously treated with an antiresorptive, or who had failed antiresorptive treatments were all given teriparatide (NCT00191425) [40, 41]. Samples for PINP assessment were prospectively collected at baseline, 1, and 6 months and analyzed by total PINP [41]. As expected, baseline PINP was significantly lower in the previous antiresorptive groups than the treatment-naive group. PINP increased from baseline in all treatment groups, with significantly greater increases noted in the treatment-naive group than the previous antiresorptive groups at 1 month. However, there were no significant differences in PINP concentrations between the treatment-naive and the previous antiresorptive groups at 6 months $[40,41]$. Overall, teriparatide increased biochemical markers of bone formation in postmenopausal women with established osteoporosis, regardless of previous long-term exposure to antiresorptive therapies [40, 41].

PINP was also evaluated in a prospective, open-label, nonrandomized study of 59 postmenopausal women with osteoporosis previously treated with raloxifene or with alendronate for at least 18 months, and then switched to teriparatide [42]. Intact PINP assessments were prospectively collected at baseline, 1, 3, 6, and 12 months of teriparatide treatment. Consistent with alendronate being a more potent inhibitor of bone turnover than raloxifene, baseline PINP
Fig. 4 Changes in intact PINP in a trial of Japanese patients with osteoporosis randomized to teriparatide or to placebo. Median change from baseline in PINP. $P I N P$ intact procollagen type I N propeptide, $I Q R$ interquartile range. ${ }^{*} p<0.001$ for within-group comparisons. The I-bars represent interquartile ranges $(25$ th, 75 th percentile). Adapted from Tsujimoto and colleagues [39]

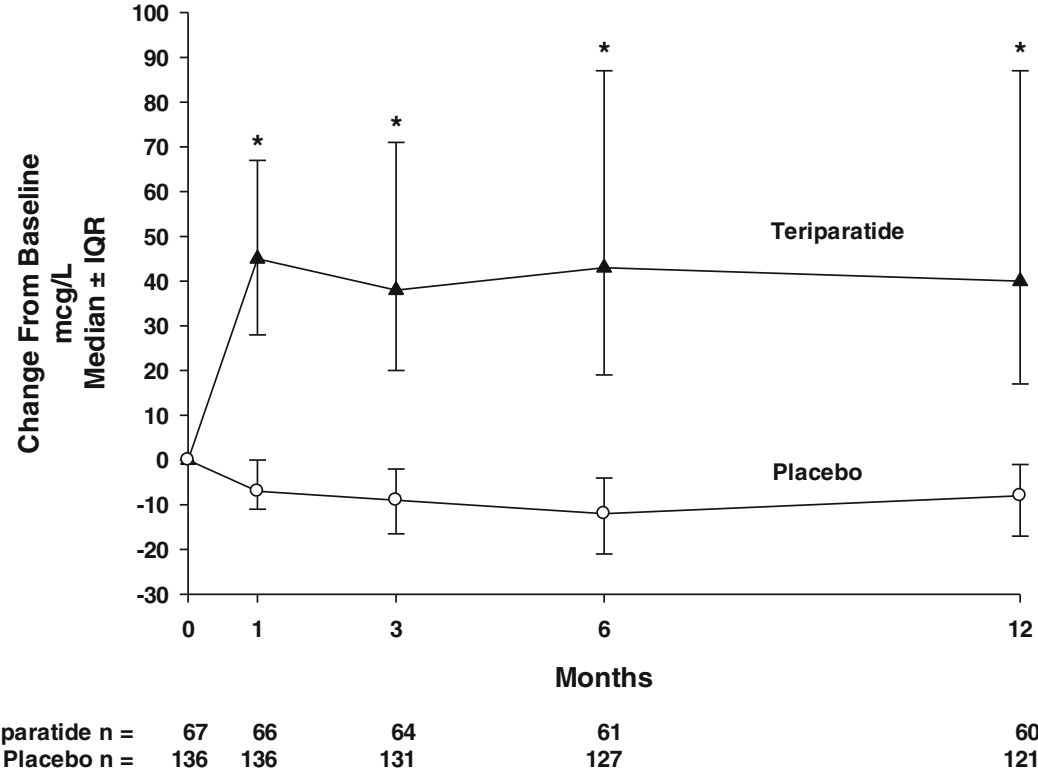


concentrations were lower in the alendronate group compared with the raloxifene group. During the treatment with teriparatide, statistically significant increases in PINP concentration from baseline occurred in both groups, but with a significantly smaller increase in the first month observed in the alendronate group than in the raloxifene group. No statistically significant difference between treatment groups occurred at subsequent time points [42]. This study included serial osteocalcin assessments. During teriparatide treatment, this marker shows changes similar to those of PINP.

In another study of patients with prior bisphosphonate exposure (alendronate, risedronate, etidronate, or pamidronate), total PINP was lower at baseline $(p=0.010)$, at 3 months, and at 6 months during teriparatide treatment in 38 patients with prior bisphosphonate exposure compared with 14 patients without prior bisphosphonate exposure [43]. These findings suggest that previous treatment with the potent antiresorptive drug alendronate appears to delay the PINP response to teriparatide, but with continued teriparatide treatment, PINP increases are observed [42, 43].

PINP response to teriparatide may be impacted differently by different bisphosphonates. For example, in the OPTAMIZE study, patients treated with alendronate or risedronate for at least 24 months were switched to teriparatide for 12 months of treatment. In the prior risedronate group, the PINP increase was significantly greater after 3 months of teriparatide than in the prior alendronate group (NCT00130403) [44].

An open-label study of 137 osteoporosis drug-naive postmenopausal women with osteoporosis, randomized to treatment with either teriparatide alone or to teriparatide plus raloxifene, evaluated intact PINP at baseline, 1, 3, and 6 months of treatment to determine the effect of the concomitant use of teriparatide with raloxifene on serum PINP concentration (NCT00046137) [45]. PINP significantly increased in both treatment groups similarly, demonstrating concomitant use of teriparatide with raloxifene resulted in a typical anabolic PINP response [45].

In regard to concomitant use of teriparatide with alendronate or with raloxifene, a prospective, open-label study of 198 postmenopausal women, with osteoporosis who were previously treated with raloxifene or alendronate for at least 18 months, randomized patients to add teriparatide and continue their antiresorptive drugs or to switch to teriparatide alone ("add" vs. "switch") (NCT00079924) [46]. Total PINP assessments were prospectively collected at baseline, 1, 3, 6, 12 , and 18 months of treatment. In all groups, PINP increased significantly from baseline during teriparatide treatment. However, the increases were smaller in the groups that added teriparatide to ongoing antiresorptive therapy compared with the groups that switched to teriparatide. In the alendronate stratum at 6 months, median PINP increases from baseline were $64 \% v s .401 \%(p<0.001)$ in the add $v s$. switch groups, respectively. In the raloxifene stratum at 6 months, median PINP increases from baseline were $131 \%$ vs. $259 \%$ $(p<0.001)$ in the add vs. switch groups, respectively [46]. These differences in PINP for add vs. switch groups demonstrate relatively greater bone formation with the switch approach, although the switch approach was also associated with greater bone resorption [46].

PINP monitoring may also find application in the management of patients with glucocorticoid-induced osteoporosis. For patients treated with chronic systemic glucocorticoids, the skeletal defect at the tissue level is impaired bone formation [47]. In a randomized, double-blind, active comparator trial of 428 men and women with glucocorticoid-induced osteoporosis (prednisone equivalent $5 \mathrm{mg}$ /day or more for at least 3 months and BMD T-score at spine or hip of -2 or less, or -1 plus a history of a fragility fracture), intact PINP assessments were prospectively collected at baseline, 1, 6, and 18 months of treatment with teriparatide or alendronate (NCT00051558) [48-52]. In the teriparatide group, PINP concentration was significantly increased $(p<0.001)$ by 1 month and peaked at 6 months, with an observed median $69.8 \%$ increase [48]. Notably, PINP concentrations remained above baseline during the 36 months of treatment [49].

From the same glucocorticoid-induced osteoporosis trial, PINP data for 83 male patients have been reported. Intact PINP serum concentration increased significantly during teriparatide treatment, peaked at 1 to 6 months, and remained above baseline at 18 months [51]. In another study of glucocorticoid-induced osteoporosis in 92 men, the typical expected increase in intact PINP during teriparatide treatment was observed, which was statistically significant as compared with risedronate $(p<0.001)$ [53]. These results confirm an increase in PINP can be expected in males with glucocorticoid-induced osteoporosis treated with teriparatide (NCT00503399) [53].

Also from the glucocorticoid-induced osteoporosis trial described above, PINP data for 67 premenopausal women have been reported (NCT00051558) [53]. During treatment with teriparatide, PINP increased from baseline at all time points, with peak increases observed at 1 to 6 months [53]. Similarly, increases in PINP were also noted in a study of 21 premenopausal women with unexplained fragility fractures or low BMD treated with teriparatide (NCT01440803) [54]. Serum PINP increased at 1 month, was $150 \%$ above baseline at 6 months, and declined toward baseline at 18- and 24month assessments [54]. These results confirm an increase in PINP can be expected in premenopausal females treated with teriparatide.

The early onset of PINP response to teriparatide was assessed in 15 osteopenic postmenopausal women treated with daily SC teriparatide $20 \mathrm{mcg} /$ day [55]. Intact PINP concentrations increased by $8.2 \%$ after 2 days of treatment and by $111 \%$ after 28 days of teriparatide treatment, with all 
observed PINP increases significant compared with baseline $(p<0.0001)$ [55]. This study showed approximately similar increases in PINP, PICP, and osteocalcin during 28 days of teriparatide treatment, but showed a smaller increase in bone ALP [55]. Following cessation of treatment at 28 days, concentrations of bone formation markers decreased to within $20 \%$ of baseline values by day 56 [55].

Relationship of PINP concentration to bone formation during teriparatide treatment

A Phase 4 clinical trial of ten postmenopausal women with osteoporosis treated with teriparatide showed a typical increase in intact PINP (NCT00259298) [56]. Increase from baseline in serum PINP concentrations at 3 and 18 months of treatment were correlated with an increase from baseline in skeletal uptake of radiopharmaceutical technetium-99 m methylene diphosphonate on a bone scan at the same visit ( $r=0.60$ at 3 months, $r=0.78$ at 18 months) [56].

In an active comparator study of 69 postmenopausal women with osteoporosis, those patients treated with teriparatide again showed a typical increase in intact PINP (NCT00927186) [57]. Additionally, the correlation between 6-month PINP concentration and bone formation, defined by the mineralizing surface of trans-iliac bone biopsies, was high $(r=0.85)$, confirming PINP concentration is related to bone formation at the bone tissue level [57]. These data demonstrate PINP concentration is strongly related to skeletal bone formation as assessed by bone scan and by trans-iliac bone biopsy.

\section{Relationship of early change in PINP concentration to other outcomes during teriparatide treatment}

Several teriparatide studies have shown statistically significant correlations between an early change in PINP concentration and a later increase in BMD during teriparatide treatment $[22,23,35,39,40,57,58]$. In general, the best correlations were obtained between change in PINP concentration at 1 month and later percent increase in lumbar spine BMD. For example, the relationship between the 1-month change in PINP vs. 12-month percent change in lumbar spine BMD is shown in Fig. 5 [39]. Note that nearly complete separation of patients treated with teriparatide from patients treated with placebo was achieved with these biomarkers. Using Spearman correlation analysis, the highest correlation coefficient value between the bone turnover marker and BMD response was observed between the change in PINP concentration at 1month and the 12-month lumbar spine BMD percent change $(r=0.76 ; p<0.010)$ [39]. These data support early increases in bone formation during teriparatide treatment, reflected by increases in PINP concentration, predict later increases in BMD.
Teriparatide studies have shown statistically significant correlations between early PINP change and later percent increases in bone strength at the spine as determined by finite element analysis. In the teriparatide vs. alendronate active comparator study described above, postmenopausal women with osteoporosis were randomized to teriparatide $20 \mathrm{mcg} /$ day or alendronate $10 \mathrm{mg} /$ day in a double-blind fashion [35]. Quantitative computed tomography scans with finite element modeling of the L3 vertebra were performed in a subset of 21 teriparatide patients at baseline and 18 months [4]. Pearson correlation coefficients were calculated for log-transformed changes from baseline in biochemical markers of bone turnover at various times and compared with changes from baseline in vertebral strength parameters at 18 months. The best predictor of 18-month vertebral strength increases was PINP increments at 1 month in teriparatide-treated patients. The correlation coefficients for vertebral compressive stiffness and volumetric BMD were 0.45 and 0.51 , respectively, with $p$ values $<0.05[4,23]$. In another study, 1- and 3-month total PINP increases vs. baseline were similarly correlated with 18month percent increase in spine strength as assessed by finite element analysis in four different groups (NCT 00079924) [46]. Additional analysis of the correlation between increases in PINP and bone strength was reported in males with glucocorticoid-induced osteoporosis [52]. In this study, changes in intact PINP at 3 months were correlated with change in finite element analysis strength increments in the spine to anterior bending $(r=0.422)$, axial compression $(r=$ $0.516)$, and axial torsion $(r=0.496)$ assessed at 6 and 18 months of treatment [52]. These findings support a correlation between early changes in serum PINP and later increases in spine BMD and strength in patients treated with teriparatide.

Data to fully assess the relationship of early PINP change with fracture risk reduction are not available, since the large Phase 3 teriparatide fracture trial included PINP assessments in only a subset of the patients $[22,23]$. However, the significant relationship between early change in PINP and later increase in BMD described above, along with the significant relationship between increase in BMD and fracture risk reduction during teriparatide treatment [24], provide indirect evidence for a relationship between PINP change and fracture risk reduction.

In the randomized, double-blind, active comparator trial of 428 men and women with glucocorticoid-induced osteoporosis described above, there was a significant reduction in vertebral fracture in the teriparatide vs. alendronate group [48, 49]. A subset of patients from both treatment groups in the trial had PINP assessments. The incidence of vertebral fractures in the subset of patients with PINP assessments was similar to the incidence of vertebral fractures in the overall study population (Fig 6). Because most patients in the teriparatide group had increases in PINP $>10 \mathrm{mcg} / \mathrm{L}$ and most 
Fig. 5 Correlation between procollagen type I N propeptide $(P I N P)$ change from baseline to 1 month $(\mathrm{mcg} / \mathrm{L})$ and percent change in lumbar spine bone mineral density $(B M D)$ from baseline to 12 months in a trial of Japanese patients with osteoporosis randomized to teriparatide or to placebo. A vertical line represents a PINP increase of $10 \mathrm{mcg} / \mathrm{L}$, and a horizontal line represents a lumbar spine BMD increase of $3 \%$. Adapted from Tsujimoto and colleagues [39]

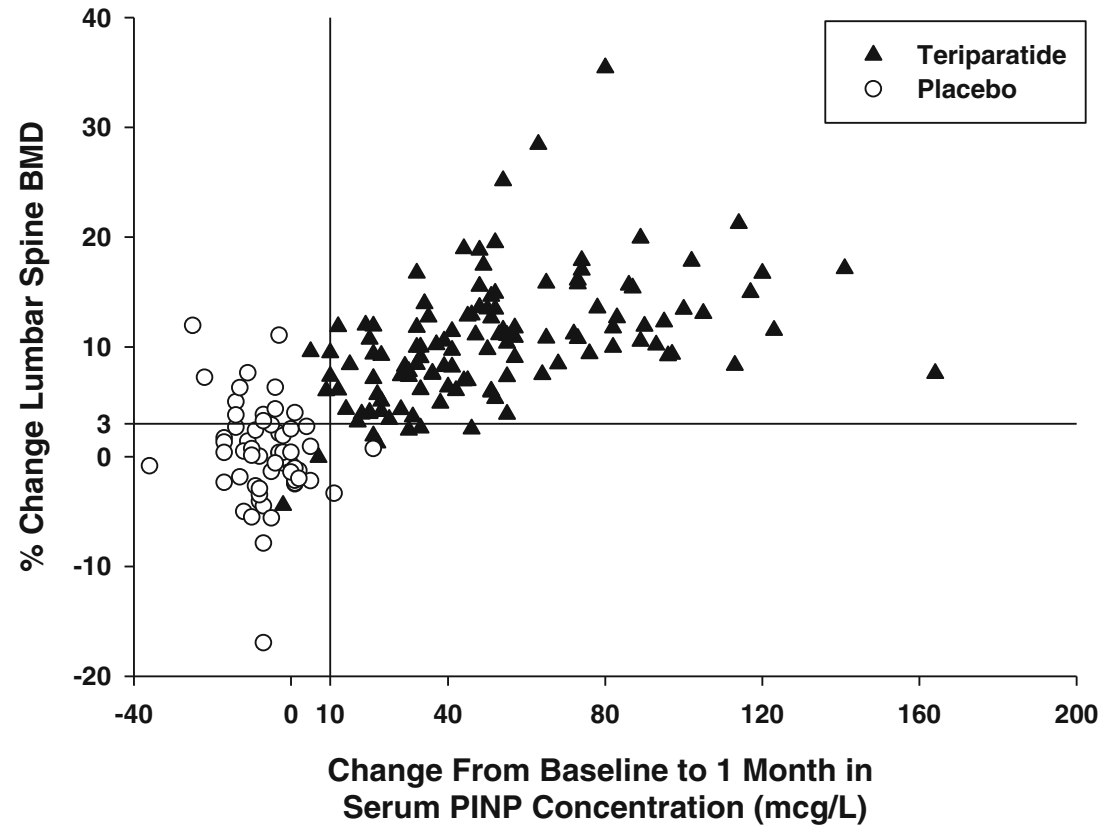

patients in the alendronate group did not, the incidence of vertebral fractures in the subgroup of patients with vs. without PINP increase from baseline $>10 \mathrm{mcg} / \mathrm{L}$ was also similar to the overall incidence (Fig. 6).

\section{PINP response in individual patients}

The intact PINP assay label references a least significant change (LSC) for PINP of $21 \%[19,59]$. However, this $21 \%$ definition of LSC for PINP during teriparatide treatment is problematic because many patients treated with teriparatide have been previously treated with potent antiresorptive drugs such as alendronate, and as noted above, these patients often have a relatively low baseline PINP concentration. For example, a typical patient treated with alendronate might have a baseline intact serum PINP concentration of $19 \mathrm{mcg} / \mathrm{L}$. In such a patient, use of the $21 \%$ cut point (LSC) would lead to the conclusion that an increase in PINP to $23 \mathrm{mcg} / \mathrm{L} \mathrm{(a} 21 \%$ increase from baseline) represents a significant increase in PINP even though this is obviously a small increment and below what would be considered a meaningful change. To overcome this difficulty, Eastell and colleagues established an absolute change cut point by using the typical mean PINP concentration of $48 \mathrm{mcg} / \mathrm{L}$ in untreated postmenopausal women [19] and multiplying the value by 0.21 (LSC) to arrive at a LSC of $>10 \mathrm{mcg} / \mathrm{L}$ [17]. Absolute change in PINP is simple to calculate and is not subject to the percentage difficulties that arise in patients with very low baseline PINP concentrations [17]. Eastell and colleagues [17] proposed an algorithm for using the observed change in PINP serum concentration to monitor patients treated with teriparatide. This algorithm was tested and slightly modified by Krege and colleagues [60] and then evaluated in detail once again by Tsujimoto and colleagues [39]. An illustration of this simplified approach to using serum PINP concentration to monitor patients treated with teriparatide is provided in Fig. 7.

A prospective Phase 3, double-blind, placebo-controlled clinical trial included prospectively defined intact PINP analyses at baseline, 1, 3, 6, and 12 months to assess response rates in individuals treated with placebo or teriparatide [38, 39] (NCT00433160). In the placebo group, 2 out of 66 patients

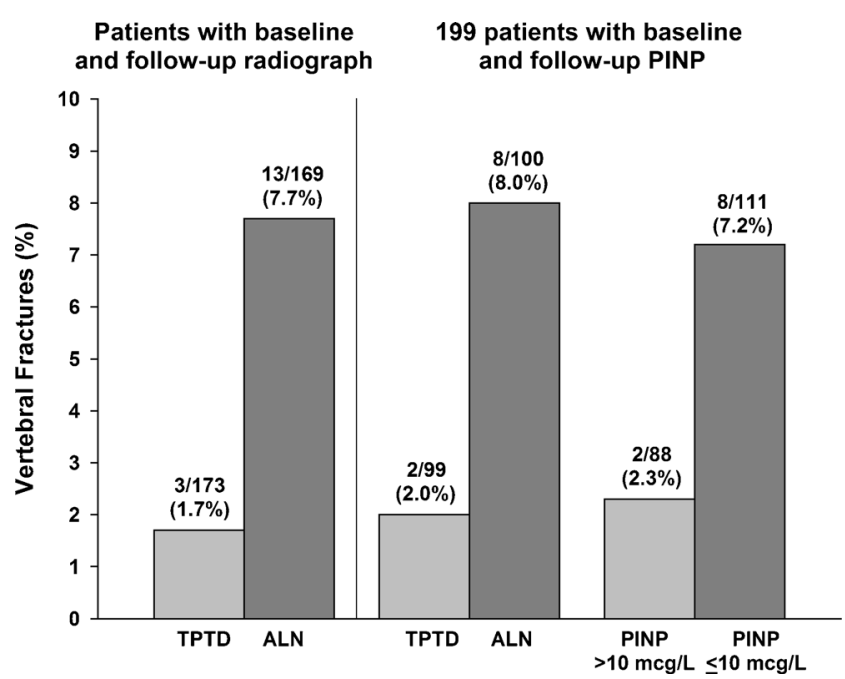

Fig. 6 Vertebral fracture outcomes at 36 months from a trial of glucocorticoid-induced osteoporosis patients randomized to alendronate or to teriparatide. The left side of the figure represents data by treatment group assignment. The right side of the figure represents data for a subset of patients $(N=199)$ who had PINP assessments at baseline, 1 , and 6 months, designating PINP increment from baseline $>10 \mathrm{mcg} / \mathrm{L}$ at 1 or 6 months. TPTD teriparatide, ALN alendronate, PINP intact procollagen type I N propeptide. Adapted from Saag and colleagues [49] 


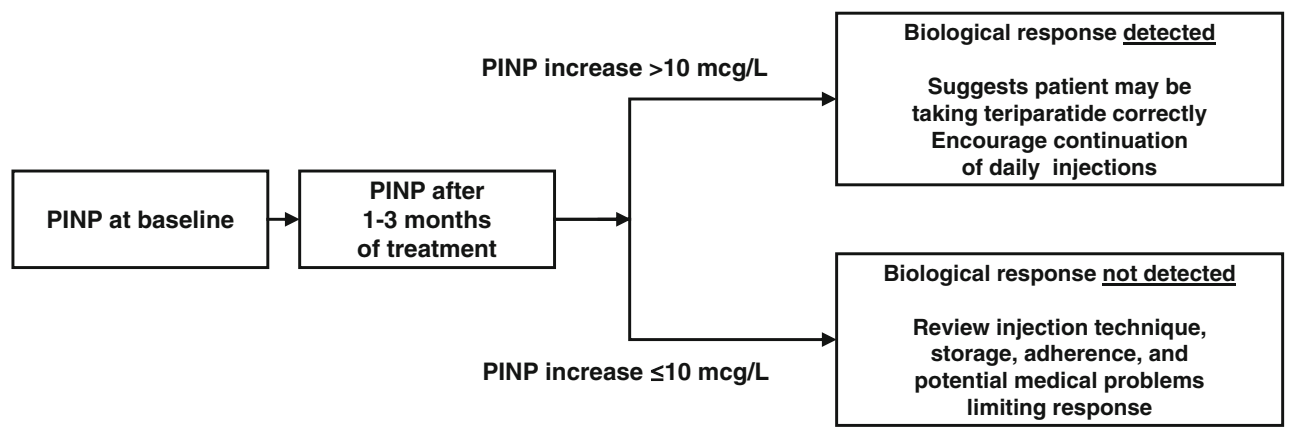

Fig. 7 Simplified approach to using PINP to assess osteoporosis patients treated with teriparatide. PINP is a biological response marker indicating whether bone formation has increased between baseline and follow-up measurements in a patient treated with teriparatide. A biological response marker is not a surrogate marker of efficacy, which would predict future outcomes like fracture. PINP intact procollagen type I N propeptide had PINP increases $>10 \mathrm{mcg} / \mathrm{L}$ at 1 or 3 months, indicating a false-positive rate of $3 \%$ when employing the monitoring algorithm. Among the 136 patients in the teriparatide $20 \mathrm{mcg} /$ day group, 129 patients had PINP increases $>10 \mathrm{mcg} / \mathrm{L}$ at 1 or 3 months, and 7 patients did not. Among these seven patients, three were noncompliant and four were compliant. The absence of an increase in PINP $>10 \mathrm{mcg} / \mathrm{L}$ at 1 or 3 months in 4 out of 136 compliant patients indicates a false-negative rate of $3 \%$ when using the monitoring algorithm [39]. Table 2 summarizes the findings from Tsujimoto and colleagues [39], defining a significant increase in PINP as an increase $>10 \mathrm{mcg} / \mathrm{L}$ at 1 or 3 months and a significant increase in lumbar spine BMD as an increase $>3 \%$ at 12 months. These results demonstrate that clinically useful complementary information may potentially be gained from monitoring both PINP and BMD during osteoanabolic therapy.

Defining significant responses in this same manner, Table 3 presents potential patient observations and health care professional responses illustrating the potential use of PINP monitoring to enhance teriparatide therapy for individual patients.

PINP response during teriparatide therapy has been evaluated in additional clinical trials in which PINP monitoring was prospectively defined $[7,17,56]$. In drug-naive postmenopausal women with osteoporosis, the proportion of patients with increases in intact PINP $>10 \mathrm{mcg} / \mathrm{L}$ was $85 \%$ at 1 month and $87 \%$ at 3 months [17]. In postmenopausal women with osteoporosis previously treated with raloxifene for at least 18 months and then switched to teriparatide, the proportion of patients with increases in intact PINP $>10 \mathrm{mcg} / \mathrm{L}$ was $96 \%$ at 1 month and was $92 \%$ at 3 months [17]. Yet, in postmenopausal women with osteoporosis previously treated with alendronate for at least 18 months and then switched to teriparatide, the proportion of patients with increases in intact PINP $>10 \mathrm{mcg} / \mathrm{L}$ was $79 \%$ at 1 month and was $97 \%$ at 3 months [17]. The observed delay in the rise of serum PINP concentration for the teriparatide group previously treated with alendronate may be a result of the prior potent antiresorptive therapy.
PINP response to teriparatide following previous therapies, with concurrent therapies, or with concomitant illnesses

Clinical study data provide insight into the effect of prior osteoporosis drug treatment on PINP response during teriparatide treatment. For example, the EUROFORS study included three osteoporosis treatment cohorts: (1) treatmentnaive patients, (2) patients pretreated with antiresorptive drugs, and (3) patients pretreated with antiresorptive drugs with inadequate clinical outcome [40, 41]. Total PINP responses $>10 \mathrm{mcg} / \mathrm{L}$ were similar in these groups [60]. Overall, the percentage of patients achieving a PINP increase $>10 \mathrm{mcg} / \mathrm{L}$ in the EUROFORS study was $83 \%(613 / 736)$ at 1 month and $91 \%(630 / 689)$ at 6 months [60]. Among the 110 patients with 1 -month PINP increases $\leq 10 \mathrm{mcg} / \mathrm{L}, 88$ (80\%) had 6-month PINP increases $>10$ mcg/l [60].

PINP response to teriparatide in patients treated with other concomitant osteoporosis medications was evaluated in a recent clinical trial including patients previously treated for at least 18 months with alendronate or raloxifene (NCT000799924) [61]. These patients were randomized either to switch to teriparatide or to add teriparatide to ongoing alendronate or raloxifene [61]. Defining a PINP response as an intact PINP increment $>10 \mathrm{mcg} / \mathrm{L}$ at 1 or 3 months, the response rates (previously unpublished data) are shown in Table 4 [61].

A study of the combination of teriparatide plus denosumab has recently been published [62]. With this treatment

Table 2 Response of PINP and BMD during osteoanabolic therapy [39]

\begin{tabular}{lll}
\hline Treatment group & PINP responder $^{\mathrm{a}}$ & BMD responder $^{\mathrm{b}}$ \\
\hline Teriparatide & $95 \%$ & $94 \%$ \\
Placebo & $3 \%$ & $20 \%$
\end{tabular}

$B M D$ bone mineral density, PINP procollagen type I N propeptide

${ }^{\mathrm{a}}>10 \mathrm{mcg} / \mathrm{L}$ increase in serum PINP concentration at 1 or 3 months

b $>3 \%$ increase in BMD at 12 months 
Table 3 Hypothetical clinical scenarios, with potential clinical responses, and relevant data from Tsujimoto and colleagues [39]

\begin{tabular}{|c|c|c|}
\hline Hypothetical clinical scenario & Hypothetical clinical response & Data to support clinical response [39] \\
\hline $\begin{array}{l}\text { No increase in PINP, patient not taking } \\
\text { teriparatide or not storing or injecting } \\
\text { the medication properly }\end{array}$ & $\begin{array}{l}\text { Patient provided assistance to acquire } \\
\text { teriparatide or education regarding } \\
\text { storage and administration }\end{array}$ & $\begin{array}{l}\text { In the placebo group, } 97 \% \text { of patients had no significant PINF } \\
\text { response. In the teriparatide group, three noncompliant } \\
\text { patients had no significant PINP response. }\end{array}$ \\
\hline $\begin{array}{l}\text { Increase in PINP, patient taking } \\
\text { teriparatide according to label }\end{array}$ & Patient encouraged to continue with therapy & $\begin{array}{l}\text { In the teriparatide group, } 95 \% \text { had significant PINP response } \\
\text { (a total of seven patients had no significant PINP response, } \\
\text { with three of these patients not being compliant with } \\
\text { treatment). }\end{array}$ \\
\hline $\begin{array}{l}\text { No increase in PINP, patient taking } \\
\text { teriparatide properly, no early increase } \\
\text { in BMD }\end{array}$ & Patient switched to alternative treatment & $\begin{array}{l}\text { In the teriparatide group, one compliant patient had no } \\
\text { significant PINP or lumbar spine BMD response and was a } \\
\text { nonresponder. }\end{array}$ \\
\hline $\begin{array}{l}\text { No increase in PINP, injecting and storing } \\
\text { teriparatide properly, BMD increases }\end{array}$ & Patient encouraged to continue with therapy & $\begin{array}{l}\text { In the teriparatide group, three patients with PINP increases of } \\
5,9 \text {, and } 10 \mu \mathrm{g} / 1 \text { had significant lumbar spine BMD } \\
\text { responses at } 6 \text { months. }\end{array}$ \\
\hline
\end{tabular}

These scenarios assume that a baseline and post-teriparatide treatment PINP sample is obtained. A significant increase in PINP was defined as an increase from baseline in PINP $>10 \mathrm{mcg} / \mathrm{L}$ at 1 or 3 months, and a significant increase in lumbar spine BMD was defined as an increase from baseline $>3 \%$

$B M D$ bone mineral density, PINP procollagen type I N propeptide

combination, intact PINP does not appear to increase, but rather PINP shows a significant decrease from baseline. This study suggests that an increase in PINP should not be anticipated to occur with combination teriparatide plus denosumab treatment [62].

PINP response to teriparatide in patients with other chronic medical illnesses has been evaluated. PINP was collected in a randomized, double-blind trial of men and women with glucocorticoid-induced osteoporosis (NCT00051558) [47-49]. These individuals had various baseline conditions for which they were treated with glucocorticoids including rheumatoid arthritis, systemic lupus erythematosis, polymyalgia rheumatica, vasculitis, respiratory disorders, and inflammatory bowel disease. In these medically complex individuals treated with teriparatide, glucocorticoids, and a variety of other concomitant medications, the proportion of individuals with increases in intact PINP $>10 \mathrm{mcg} / \mathrm{L}$ at 1 or 6 months was $88 \%$ [63].

\section{Discussion}

As highlighted in this review, PINP may be most useful as a biological response marker. In clinical trials, PINP testing usually detects a biological response to teriparatide treatment and a lack of biological response to placebo. While the data provided in this review are, in general, supportive that an increase in PINP during teriparatide treatment is associated with increases in BMD and bone strength, increases in PINP during teriparatide treatment have not been directly validated to predict fracture risk reduction. This proof is not yet available because PINP monitoring was only performed on stored samples from a subset of patients enrolled in the Phase 3 teriparatide fracture trial [17].
Using PINP as a biological response marker during teriparatide treatment for osteoporosis has some limitations. Results of the PINP assay should be used in conjunction with other pertinent clinical information to make diagnostic and therapeutic decisions. Most importantly, PINP testing does not replace BMD testing. Rather, as highlighted in this review, the results of PINP monitoring may be complementary to those of BMD testing [11, 27, 64]. The PINP assay is not recommended for use as a screening procedure to detect the presence of osteoporosis in the general population [19]. And, as yet, the evidence base that biochemical markers of bone turnover can be used in the assessment of fracture risk in individual patients is limited [17, 27]. There is a chance for both false-positive and false-negative results with all laboratory tests, and this is a potential limitation with PINP testing. The clinical trials reviewed herein have demonstrated that a majority of, but not all, patients taking teriparatide will have an increase in PINP concentration from baseline, and PINP may increase in some patients taking placebo. This problem might be more of an issue in clinical practice than in the setting of clinical trials. To reduce variability of PINP assessments in clinical practice,

Table 4 PINP response by treatment group in Cosman and colleagues [61]

\begin{tabular}{ll}
\hline Treatment group & PINP response $^{\mathrm{a}}, N(\%)$ \\
\hline Alendronate plus teriparatide & $40 / 52(77 \%)$ \\
Raloxifene plus teriparatide & $45 / 47(96 \%)$ \\
Switched from alendronate or raloxifene & $97 / 99(98 \%)$ \\
$\quad$ to teriparatide & \\
\hline
\end{tabular}

PINP procollagen type I N propeptide

${ }^{\text {a }} \mathrm{PINP}$ increment $>10 \mathrm{mcg} / \mathrm{L}$ at 1 or 3 months 
it might be possible to store the baseline sample at $-20^{\circ} \mathrm{C}$ [19] and to run the baseline and follow-up serum sample as a batch.

As yet, the evidence for use of biochemical markers as a tool to improve adherence with treatment is weak. For example, a study of 596 patients treated with monthly ibandronate examined the impact of serum $\mathrm{C}$-terminal cross-linking telopeptide of type I collagen (CTX-1, a biochemical marker of bone resorption) monitoring and feedback on patient adherence [65]. The results were similar among the groups whether they received biochemical marker feedback or not, with adherence documented in $92.6 \%$ of patients given feedback and $96 \%$ of patients not given feedback [65]. However, as noted in this particular trial, participants in clinical trials tend to have such high adherence that improvements may be difficult to document. Also, while this study showed no difference in adherence, patients provided with information about their biochemical markers of bone turnover felt more informed about their osteoporosis $(p<0.001)$ and more satisfied $(p<0.010)$ than those patients not provided with information [65]. Whether testing of biochemical markers of bone turnover may improve adherence outside of the setting of clinical studies is not yet known.

In the clinical setting, biochemical markers of bone turnover may be elevated for some months after a fracture event and during the fracture healing process [66]. Some of the studies in this review included many patients with previous fracture $[24,58]$ and demonstrated correlations between early increases in PINP and later increases in BMD. Accordingly, it is possible that a recent fracture does not preclude a typical PINP response to teriparatide treatment. The question of the effect of recent fracture on the PINP response to teriparatide could potentially be addressed through post hoc analyses of appropriate clinical trials.

The IOF, the IFCC, and the National Bone and Health Alliance recommend a number of measures for optimizing the clinical use of PINP monitoring in the day-to-day care of patients with osteoporosis $[12,13,67]$. In addition to the importance of harmonization for future research of bone turnover markers, these groups recommend the standardization of bone marker sample collection procedures, reference ranges, and bone turnover marker assays in clinical laboratories [12, $13,67,68]$. Toward this end, preliminary data from an external quality assurance scheme suggest that the three major serum PINP assays provide harmonized results [68]. However, as previously discussed, total PINP provides higher values than intact PINP in the setting of impaired renal function [31, 32].

In summary, PINP is a product of the processing of type I procollagen to mature type I collagen, the most prevalent protein in the bone [19]. Quantifying PINP measures anabolic activity in the bone. Depending on geography, serum assays for measuring PINP concentration are available and approved to aid in the management of patients with postmenopausal osteoporosis. Based on advantages of PINP monitoring and extensive literature regarding the effects of various therapeutic agents on concentrations of PINP, the PINP test is recommended as the reference biochemical marker of bone formation by the IOF, IFCC, and the National Bone Health Alliance. Substantial evidence from clinical trials demonstrates PINP is a biological response marker that increases rapidly and dynamically in most teriparatide-treated patients and does not increase in most placebo-treated patients. A biological response to anabolic osteoporosis therapy may be defined as an increase in PINP concentration $>10 \mathrm{mcg} / \mathrm{L}$ from baseline. PINP monitoring may be useful for clinicians prescribing teriparatide for osteoporosis patients at high risk for fracture. Data validating the use of PINP for predicting fracture risk reduction during teriparatide treatment are not available.

PINP testing has the potential to support patients taking teriparatide as prescribed, and thereby maximize their chances for clinical benefit and positive treatment outcome.

Acknowledgments The authors would like to acknowledge Julie Sherman, an employee of Eli Lilly and Company, for assistance in preparing the manuscript figures. For assistance with data integrity verification, the authors thank Heather Murphy, Biostatistician, an employee of inVentiv Health clinical; Helen Li, Senior Statistical Programmer, an employee of inVentiv Health clinical; Abby Wu, Senior Statistical Programmer, an employee of inVentiv Health clinical; and Qiu He, Consultant Statistician, an employee of Eli Lilly and Company. Funding was provided by Eli Lilly and Company.

Conflicts of interest JH Krege is an employee and shareholder of Eli Lilly and Company. NE Lane reports no potential conflict of interest relevant to the submitted work or outside the submitted work. JM Harris is an employee of Eli Lilly and Company. PD Miller reports no potential conflict of interest relevant to the submitted work, but has received research grants from Amgen, Eli Lilly and Company, Merck, Radius, Merck Serono, Agnovus, Boehringer Ingelheim outside the submitted work, and has participated in an Amgen Scientific Advisory Board outside the submitted work.

Open AccessThis article is distributed under the terms of the Creative Commons Attribution Noncommercial License which permits any noncommercial use, distribution, and reproduction in any medium, provided the original author(s) and the source are credited.

\section{References}

1. Neer RM, Arnaud CD, Zanchetta JR et al (2001) Effect of parathyroid hormone (1-34) on fractures and bone mineral density in postmenopausal women with osteoporosis. N Engl J Med 344(19):1434-1441

2. Jiang Y, Zhao JJ, Mitlak BH, Wang O, Genant HK, Eriksen EF (2003) Recombinant human parathyroid hormone (1-34) [teriparatide] improves both cortical and cancellous bone structure. J Bone Miner Res 18(11):1932-1941

3. Lindsay R, Cosman F, Zhou H et al (2006) A novel tetracycline labeling schedule for longitudinal evaluation of the short-term effects of anabolic therapy with a single iliac crest bone biopsy: early actions of teriparatide. J Bone Miner Res 21(3):366-373 
4. Keaveny TM, Donley DW, Hoffmann PF, Mitlak BH, Glass EV, San Martin JA (2007) Effects of teriparatide and alendronate on vertebral strength as assessed by finite element modeling of QCT scans in women with osteoporosis. J Bone Miner Res 22(1):149-157

5. Graeff C, Chevalier Y, Charlebois M et al (2009) Improvements in vertebral body strength under teriparatide treatment assessed in vivo by finite element analysis: results from the EUROFORS study. J Bone Miner Res 24(10):1672-1680

6. Prevrhal S, Krege JH, Chen P, Genant H, Black DM (2009) Teriparatide vertebral fracture risk reduction determined by quantitative and qualitative radiographic assessment. Curr Med Res Opin 25(4):921-928

7. Krege JH, Wan X (2012) Teriparatide and the risk of nonvertebral fractures in women with postmenopausal osteoporosis. Bone 50(1): 161-164

8. FORTEO $^{\circledR}$ (teriparatide [rDNA origin] injection) for subcutaneous USPDI. http://dailymed.nlm.nih.gov/dailymed/lookup.cfm?setid= aae667c5-381f-4f92-93df-2ed6158d07b0. Accessed 4 June 2013

9. Curtis JR, Cai Q, Wade SW et al (2013) Osteoporosis medication adherence: physician perceptions vs. patients' utilization. Bone $55(1): 1-6$

10. International Society of Clinical Densitometry. 2007 Official positions and pediatric official positions of the International Society for Clinical Densitometry, West Hartford, CT. http://www.ISCD.org. Accessed 20 September 2012

11. Bonnick SL, Lee Shulman L (2006) Monitoring osteoporosis therapy: bone mineral density, bone turnover markers, or both? Am J Med 119(4A):25S-31S

12. Vasikaran S, Eastell R, Bruyère $O$ et al (2011) Markers of bone turnover for the prediction of fracture risk and monitoring of osteoporosis treatment: a need for international reference standards. Osteoporos Int 22(2):391-420

13. Bauer D, Krege J, Lane N et al (2012) National bone health alliance bone turnover marker project: current practices and the need for US harmonization, standardization, and common reference ranges. Osteoporos Int 23(10):2425-2433

14. Civitelli R, Armamento-Villareal R, Napoli N (2010) Bone turnover markers: understanding their value in clinical trials and clinical practice. Osteoporos Int 20(6):843-851

15. Delmas PD, Vrijens B, Roux C et al (2013) A reinforcement message based on bone turnover marker response influences long-term persistence with risedronate in osteoporosis: the IMPACT study. J Bone Miner Res 18:S374

16. International Osteoporosis Foundation. The adherence gap: why osteoporosis patients don't continue with treatment. http://www. iofbonehealth.org/sites/default/files/PDFs/adherence_gap_report_ 2005.pdf. Accessed 22 May 2013

17. Eastell R, Krege JH, Chen P, Glass EV, Reginster JY (2006) Development of an algorithm for using PINP to monitor treatment of patients with teriparatide. Curr Med Res Opin 22(1):61-66

18. Terreni A, Pezzati P (2012) Biochemical markers in the follow-up of the osteoporotic patients. Clin Cases Min Bone Metab 9(2):80-84

19. Orion Diagnostica. UniQ ${ }^{\mathrm{TM}}$ PINP RIA: intact N-terminal propeptide of type I procollagen radioimmunoassay kit. January 2008. Catalogue Number 67034. http://us.idsplc.com/en-us/products/ product.php?id=6309. Accessed 3 June 2013

20. Lodish H, Berk A, Zipursky SL, Matsudaira P, Baltimore D, Darnell J (2000) Collagen: the fibrous proteins of the matrix. In: Molecular cell biology, 4th edn. WH Freeman, New York, Table 22-3. http://www. ncbi.nlm.nih.gov/books/NBK21475/. Accessed 2 December 2013

21. Melkko J, Kauppilla S, Niemi S, Risteli L, Haukipuro K, Jukkola A, Risteli J (1996) Immunoassay for intact aminoterminal propeptide of human type I procollagen. Clin Chem 42(6): $947-954$

22. Chen P, Satterwhite JH, Licata AA et al (2005) Early changes in biochemical markers of bone formation predict BMD response to teriparatide in postmenopausal women with osteoporosis. J Bone Miner Res 20(6):962-970

23. Chen P, Glass EV, Krege JH. Early changes in bone turnover markers (BTMs) predict vertebral strength in teriparatide- or alendronatetreated postmenopausal women with osteoporosis [abstract]. In: ENDO 2007 Program \& Abstracts, June 2007, Endocrine Society, Toronto, Canada

24. Chen P, Miller PD, Delmas PD, Misurski DA, Krege JH (2006) Change in lumbar spine BMD and vertebral fracture risk reduction in teriparatide-treated postmenopausal women with osteoporosis. J Bone Miner Res 21(11):1785-1790

25. Naylor K, Eastell R (2012) Bone turnover markers: use in osteoporosis. Nat Rev Rheumatol 8(7):379-389

26. United States Department of Health and Human Services, Food and Drug Administration, Center for Drug Evaluation and Research (CDER). Guidance for industry: qualification process for drug development tools. Draft guidance, October 2010. http:/www.fda.gov/ downloads/Drugs/GuidanceComplianceRegulatoryInformation/ Guidances/UC<230597.pdf. Accessed 22 May 2013

27. Miller PD (2012) Bone strength and surrogate markers: the 1st, 2nd, and 3rd fiddle. J Bone Miner Res 27(8):1623-1626

28. Immunodiagnosticsystems. Fully automated intact PINP. http://www. pantec.it/files/IDS-iSYS-Intact-PINP-IS-4000.pdf. Accessed 4 June 2013

29. Roche Diagnostics. http://www.roche.com/products/product-list. $\mathrm{htm}$ ?region=us\&type $=$ diagnostics\&id=24. Accessed 22 May 2013

30. USCN Life Science. ELISA kit. http://www.uscnk.us/elisa/ELISAKit-for-Human-Procollagen-I-N-Terminal-Propeptide-PINP-1354. htm. Accessed 3 June 2013

31. Koivula M, Risteli L, Risteli J (2012) Measurement of aminoterminal propeptide of type 1 procollagen (P1NP) in serum. Clin Biochem 45(12):920-927

32. Cavalier E, Lukas P, Ferrante N, Rousselle O, Carlisi A, Delanaye P (2013) Difference between total and intact assays for N-terminal propeptide of type I procollagen (PINP) Determination in renal impaired patients. OC 29. In: EuroMEdLab 2013 - Scientific sessions. 20th IFCC-EFLM European congress of clinical chemistry and laboratory medicine (EuroMedLab) and 45th congress of the Italian society of clinical biochemistry and clinical molecular biology (SIBioC), 19-23 May 2013, Milan, Italy. Biochimica Clinica 37: S42. http://www.sibioc.it/upload/bc/37/SS/915_7-83_Scientific Sessions.pdf. Accessed 22 May 2013

33. Insogna K, Simpson C (2010) A new automated method for measuring intact amino-terminal propeptide of type 1 procollagen (PINP). J Bone Miner Res 25(Suppl 1). http://www.asbmr.org/Meetings/ AnnualMeeting/AbstractDetail.aspx?aid=1e5dd592-c08c-4692a19b-6a7927a93888. Accessed 13 May 2013

34. Richardson J, Griffiths K, Bennett AH, Garrity ML, Barnes AK. A new automated intact amino-terminal propeptide of type I procollagen (PINP) assay* on the IDS-iSYS analyser. http://www. idsplc.com/z_includes/z_assets/asset_file.php?id=9237. Accessed 5 August 2013

35. McClung M, San Martin J, Miller PD et al (2005) Opposite bone remodeling effects of teriparatide and alendronate in increasing bone mass. Arch Intern Med 165(15):1762-1768

36. Anastasilakis AD, Goulis DG, Polyzos SA et al (2008) Head to head comparison of risedronate vs. teriparatide on bone turnover markers in women with postmenopausal osteoporosis: a randomized trial. Int J Clin Pract 62(6):919-924

37. Recker RR, Marin F, Ish-Shalom S et al (2009) Comparative effects of teriparatide and strontium ranelate on bone biopsies and biochemical markers of bone turnover in postmenopausal women with osteoporosis. J Bone Miner Res 24(8):1358-1368

38. Miyauchi A, Matsumoto T, Sugimoto T, Tsujimoto M, Warner MR, Nakamura T (2010) Effects of teriparatide on bone mineral density and bone turnover markers in Japanese subjects with osteoporosis at 
high risk of fracture in a 24-month clinical study; 12-month, randomized, placebo-controlled, double-blind and 12-month open-label phases. Bone 47(3):493-502

39. Tsujimoto M, Chen P, Miyauchi A, Sowa H, Krege JH (2011) PINP as an aid for monitoring patients treated with teriparatide. Bone 48(4): 798-803

40. Blumsohn A, Marin F, Nickelsen T et al (2011) Early changes in biochemical markers of bone turnover and their relationship with bone mineral density changes after 24 months of treatment with teriparatide. Osteoporos Int 22(6):1935-1946

41. Boonen S, Marin F, Obermayer-Pietsch B et al (2008) Effects of previous antiresorptive therapy on the bone mineral density response to two years of teriparatide treatment in postmenopausal women with osteoporosis. J Clin Endocrinol Metab 93(3):852-860

42. Ettinger B, San Martin J, Crans G, Pavo I (2004) Differential effects of teriparatide on BMD after treatment with raloxifene or alendronate. J Bone Miner Res 19(5):745-751

43. Middleton ET, Steel SA, Doherty SM (2007) The effect of prior bisphosphonate exposure on the treatment response to teriparatide in clinical practice. Calcif Tissue Int 81(5):335-340

44. Miller PD, Delmas PD, Lindsay R et al (2008) Early responsiveness of women with osteoporosis to teriparatide after therapy with alendronate or risedronate. J Clin Endocrinol Metab 93:3785-3793

45. Deal C, Omizo M, Schwartz EN et al (2005) Combination teriparatide and raloxifene therapy for postmenopausal osteoporosis: results from a 6-month double-blind placebo-controlled trial. J Bone Miner Res 20(11):1905-1911

46. Cosman F, Wermers RA, Recknor C et al (2009) Effects of teriparatide in postmenopausal women with osteoporosis on prior alendronate or raloxifene: differences between stopping and continuing the antiresorptive agent. J Clin Endocrinol Metab 94(10):37723780

47. Devogelaer JP, Adler RA, Recknor C et al (2010) Baseline glucocorticoid dose and bone mineral density response with teriparatide or alendronate therapy in patients with glucocorticoid-induced osteoporosis. J Rheumatol 37(1):141-148

48. Saag KG, Shane E, Boonen $S$ et al (2007) Teriparatide or alendronate in glucocorticoid-induced osteoporosis. N Engl J Med 357(20): 2028-2039

49. Saag KG, Zanchetta JR, Devogelaer JP et al (2010) Effects of teriparatide versus alendronate for treating glucocorticoid-induced osteoporosis: thirty-six-month results of a randomized, doubleblind, controlled trial. Arthritis Rheum 60(11):3346-3355

50. Burshell AL, Moricke R, Correa-Rotte R et al (2010) Correlations between biochemical markers of bone turnover and bone density responses in patients with glucocorticoid-induced osteoporosis treated with teriparatide or alendronate. Bone 46(4):935-939

51. Langdahl BL, Marin F, Shane E et al (2009) Teriparatide versus alendronate for treating glucocorticoid-induced osteoporosis: an analysis by gender and menopausal status. Osteoporos Int 20(12):20952104

52. Farahmand P, Marin F, Hawkins F et al (2013) Early changes in biochemical markers of bone formation during teriparatide therapy correlate with improvements in vertebral strength in men with glucocorticoid-induced osteoporosis. Osteoporos Int 24:2971-2981

53. Glüer CC, Marin F, Ringe JD et al (2013) Comparative effects of teriparatide and risedronate in glucocorticoid-induced osteoporosis in men: 18-month results of the EuroGIOPs trial. J Bone Miner Res 28(6):1355-1368
54. Cohen A, Stein EM, Recker RR et al (2013) Teriparatide for idiopathic osteoporosis in premenopausal women: a pilot study. J Clin Endocrinol Metab 98(5):1971-1981, Mar 29Epub 2013

55. Glover SJ, Eastell R, McCloskey EV et al (2009) Rapid and robust response of biochemical markers of bone formation to teriparatide therapy. Bone 45(6):1503-1508

56. Moore AE, Blake GM, Taylor KA et al (2010) Assessment of regional changes in skeletal metabolism following 3 and 18 months of teriparatide treatment. J Bone Miner Res 25(5): 960-967

57. Dempster DW, Zhou H, Recker RR et al (2012) Skeletal histomorphometry in subjects on teriparatide or zoledronic acid therapy (SHOTZ) study: a randomized controlled trial. J Clin Endocrinol Metab 97(8):2799-2808

58. Niimi R, Kono T, Nishihara A et al. (2013) An algorithm using the early changes in PINP to predict the future BMD response for patients treated with daily teriparatide. Osteoporos Int 2013 Jun 29 [Epub ahead of print]. doi 10.1007/s00198-013-2426-2

59. Hannon R, Blumsohn A, Naylor K, Eastell R (1998) Response of biochemical markers of bone turnover to hormone replacement therapy: impact of biological variability. J Bone Miner Res 13(7):1124 1133

60. Krege JH, Blumsohn A, Nickelsen TA et al (2006) Testing an algorithm for using PINP to monitor treatment of patients with teriparatide (abstract). J Bone Miner Res 21(Suppl 1):S302

61. Cosman F, Keaveny TM, Kopperdahl D et al (2013) Hip and spine strength effects of adding versus switching to teriparatide in postmenopausal women with osteoporosis treated with prior alendronate or raloxifene. J Bone Miner Res 28(6):1328-1336

62. Tsai JN, Uihlein AV, Lee H et al (2013) Teriparatide and denosumab, alone or combined, in women with postmenopausal osteoporosis: the DATA study randomized trial. Lancet 382:50-56

63. Lane NE, See K, Warner MR, Krege JH (2010) Algorithm for using a bone formation marker PINP to monitor the response to teriparatide (TPTD) in patients with glucocorticoid-induced osteoporosis (GIO). Arthritis Rheum 62(Suppl 10):957

64. Funck-Brentano T, Biver E, Chopin F et al (2011) Clinical utility of serum bone turnover markers in postmenopausal osteoporosis therapy monitoring: a systematic review. Semin Arthritis Rheum 41(2): 157-169

65. Kung AW, Rachman IA, Adam JM et al (2009) Impact of bone marker feedback on adherence to once monthly ibandronate for osteoporosis among Asian postmenopausal women. Int J Rheum Dis 12(3):216-224

66. Delmas PD, Eastell R, Garnero P et al (2000) The use of biochemical markers of bone turnover in osteoporosis Committee of Scientific Advisors of the International Osteoporosis Foundation. Osteoporos Int 11(Suppl 6):S2-S17

67. National Osteoporosis Foundation (2013) Clinician's guide to prevention and treatment of osteoporosis. National Osteoporosis Foundation, Washington, DC

68. Morris H (2013) The need for standardisation of bone marker assays. SY 69. In: EuroMEdLab 2013 - scientific sessions. 20th IFCCEFLM European congress of clinical chemistry and laboratory medicine (EuroMedLab) and 45th congress of the Italian society of clinical biochemistry and clinical molecular biology (SIBioC), 2013 19-23 May, Milan, Italy. Biochimica Clinica 37:S41. http://www. sibioc.it/upload/bc/37/SS/915 7-83_Scientific_Sessions.pdf. Accessed 22 May 2013 